\title{
Using a Rapid Appraisal Approach in a Nationwide, Multisite Public Involvement Study in Scotland
}

Qualitative Health Research

Volume X Number X

Month XXXX xx-xx

(C) 2008 Sage Publications $10.1177 / 1049732308318735$ http://qhr.sagepub.com hosted at

http://online.sagepub.com

\author{
Neneh Rowa-Dewar \\ Wendy Ager \\ Katherine Ryan \\ Irene Hargan \\ Gill Hubbard \\ Nora Kearney \\ University of Stirling, Stirling, United Kingdom
}

\begin{abstract}
This article reflects on the use of a multisite rapid appraisal (RA) approach with reference to the (to date) largest qualitative study gathering the views of cancer and cancer care in a cross-section of the Scottish population. A series of ten RAs were conducted in ten communities across Scotland, reflecting the geographical and socioeconomic spread of the Scottish population and involving 507 members of the public, including the views of people who are often termed "hard to reach." The research method is evaluated with reference to principles of RA approaches: the inductive approach, triangulation, assessment and response, and participation. Presentation of the methods adopted in this study demonstrates the value of the rapid appraisal approach in engaging with members of the public in health-related issues, which belies the "quick and dirty" reputation of RA approaches, and offers a model for future public involvement work in health care. This makes the reflections on the method utilized particularly relevant to policy makers and researchers wishing to achieve meaningful public involvement and/or consider a method not previously used in this context.
\end{abstract}

Keywords: combined methods; data collection and management; field methods; health

E ngaging the public in the development of health services is viewed as an important, if somewhat political, imperative within current health care practice. This extends the concept of involving the public in health issues and assumes a willingness and altruism to ensure and improve the well-being of society. Furthermore, the involvement agenda within the United Kingdom National Health Service (NHS) also aims to involve patients and carers in the process of service development to make sure their views are included (Scottish Executive Health Department [SEHD], 2005).

Although strategies and directives for involving patients and carers in Scottish health policy exist (SEHD, 2000), there is little evidence of initiatives to listen to the views of other members of the general public, so that they too can influence health services research, policy, planning, and practice. Traditional ways to encourage public participation (including public meetings, patient groups, and complaints procedures) have met with limited success and there appears to be no consensus on a standard methodology for gathering the public's views about health care (for further discussion on different public involvement approaches see Oliver et al., 2004).

In contrast, rapid appraisal (RA) approaches have been successfully used for the assessment of local needs in developing (Materia et al., 1995; Paredes et al., 1997) as well as developed countries (Murray, 1999; Ong \& Humphries, 1994) Manderson and Aaby (1992) refer to the "epidemic" rise in the use of the method, though rapid appraisal methods remain peripheral to mainstream public health (Rhodes, Stimson, Fitch, Ball, \& Renton, 1999). This might be linked to the low frequency of publication of papers in peer-reviewed journals exploring this approach, despite its common application in practice (Fitch, Stimson, Rhodes, \& Poznyak, 2004) and questions raised about its methodological validity (e.g., McKeganey, 2000).

Current forms of RA techniques originated in the 1970 s as a response to the need for quick access to 
useable and relevant socioeconomic information about local needs and priorities in developing countries to develop policies and programs (Fitch et al., 2004). RA methods stem from a recognition that "those who make the recommendations and the decisions ... are often poorly informed about the realities of those living with their decisions" (Cornwall \& Guijt, 2004) and are based on the premise that a representative picture of the views, needs, and/or priorities of a local population can be derived from a smaller number of key informants (Ong \& Humphries, 1994).

Local key informants, identified as being those in the best position to understand the issues in question, are therefore selected in a purposeful manner (Murray, 1999). Involving different stakeholders and using a battery of research methods such as socioeconomic data about, and focus groups with, members of the local population during a short space of time are thought to provide a cost-effective and less time-consuming method than other traditional methods.

Although those who promote the use of RA approaches are pragmatic about the level of precision needed and acknowledge there are limits to what needs to be known (Heaver, 1992), the term "rapid" should not necessarily be taken to imply a "quick and dirty" method lacking in rigor. The inherent triangulation of sources of data and methods of data collection provides opportunities for cross-checking and validating findings throughout (Koelen, Vaandrager, \& Colomer, 2001; Rhodes et al, 1999; Tones \& Green, 2004). The cyclical process also provides the potential for members of the community to reflect on findings as they take shape, and encourages their active participation in the research process (Koelen et al., 2001).

This article is a contribution to the discussion on the methodological approaches used to meaningfully engage the public in discussions about health-related issues. It also addresses the concern that RA studies are akin to "bad science" (McKeganey, 2000) or lack reflective awareness of the limitations of the approach (Campbell, 2002). Using the example of a study we recently conducted to gather the views of members of the Scottish public in relation to cancer and cancer care, the process of rapid appraisal we undertook will be described and we will then reflect on our experience. This reflection is based on the methodological principles associated with rapid appraisal identified by Rhodes et al. (1999). Rhodes and his colleagues evaluated the World Health Organization's "rapid appraisal and response" method in relation to injection of drugs and its associated health problems. They concluded that this method effectively linked assessment with the development of effective interventions, adding to the evidence that rapid appraisal is an important public health tool. In their paper, they highlighted four methodological principles which we utilize here to reflect on our multisite study in Scotland. These four methodological prnciples are (Rhodes et al., 1999):

1. The inductive approach: "allows assessments to develop in response to practical findings as they emerge"

2. The focus on triangulation: "triangulation between multiple methods and data sources"

3. Assessment as an integral part of the response: focuses on the outcomes of appraisal and the extent to which the assessment process is "an integral part of the response"

4. Priority to participation: "priority given to participatory initiatives" within rapid appraisal

\section{Methods}

Following examples conducted across the world (Burrows, Trautman, Bijl, \& Sarankov, 1999; Dorabjee \& Samson, 2000; Needle et al., 2003), a multisite study was undertaken. Significant geographical and social variations exist within Scotland that might have an impact on the risk and outcome of many diseases. Such differences might include, for example, access to health care services for people living in rural/urban areas and higher risk and a poorer outcome for older people and for people living in deprived areas (National Health Services Scotland, Information Services Division, 2007). Areas for inclusion were therefore selected to reflect the geographical and socioeconomic spread of the Scottish population. Ten RAs were conducted with a total of 507 participants. Eight appraisals were conducted in three socioeconomically disadvantaged, three advantaged, and two mid-range geographical locations, covering all deprivation category (Depcat) scores from most affluent (1) to very deprived ( $7 ; 331$ total adult participants). Two further appraisals were undertaken with groups commonly termed as "hard to reach": children (132 participants in one advantaged, one disadvantaged, and one mid-range community), and the two largest ethnic and black minority groups in Scotland-Asian and Chinese people (44 adult participants in large urban, mixed localities). Of the 347 of the 375 adults who completed the demographic exit questionnaire, there were 126 males and 219 females aged 16 to $85+$.

Because RA is a new approach for exploring public views on cancer, the methods were piloted in two communities — one deprived and one affluent. Despite 
advertising in local newspapers, radio stations, leaflets and posters in the local area, and visits to local community groups to promote the meetings, public attendance at these meetings was poor. This method of data collection was therefore not continued in the main study but replaced by open stalls (booths) in the "hub" of each community, in addition to a well-attended meeting in a predominantly male place of work during working hours.

Data collection for the ten RAs took place during 11 months in 2005. Throughout this study we adopted ethical procedures from the Market Research Code of Conduct (Market Research Society/Social Research Association, 2005), which is applicable as it includes principles that relate specifically to eliciting the views of members of the public.

A combination of qualitative, quantitative, and participatory research methods were used as part of the RA approach to gather information, including documentary sources, individual interviews, focus groups, open meetings, and questionnaires. The rapid appraisal process is depicted in Figure 1 and includes a series of steps which we detail below.

\section{Developing a Community Profile}

Information was gathered to provide a general social and health profile of the area, including life expectancy, education level, socioeconomic status, occupation, and health status. Sources included census data from local councils, health and well-being profile constituency data from National Health Services and incident and mortality cancer data from Information Services Division (National Health Services Scotland, 2007).

\section{Identifying and Interviewing Key Informants}

Up to ten key informants were identified in each area, usually including the lead cancer clinician, political representatives (members of Parliament and the Scottish Parliament, specialist cancer nurses; local health professionals (general practitioner, district nurse, community nurses), and local social care/community workers. A "snowballing" procedure was also used to identify less public but equally key members of the community by asking both key informants and people participating in the focus groups (MacDougall \& Fudge, 2001).

\section{Holding Open Stalls}

Open stalls were held at the hub of each community (shopping center, library, and so forth), as identified by
Figure 1

\section{Rapid Appraisal Process}

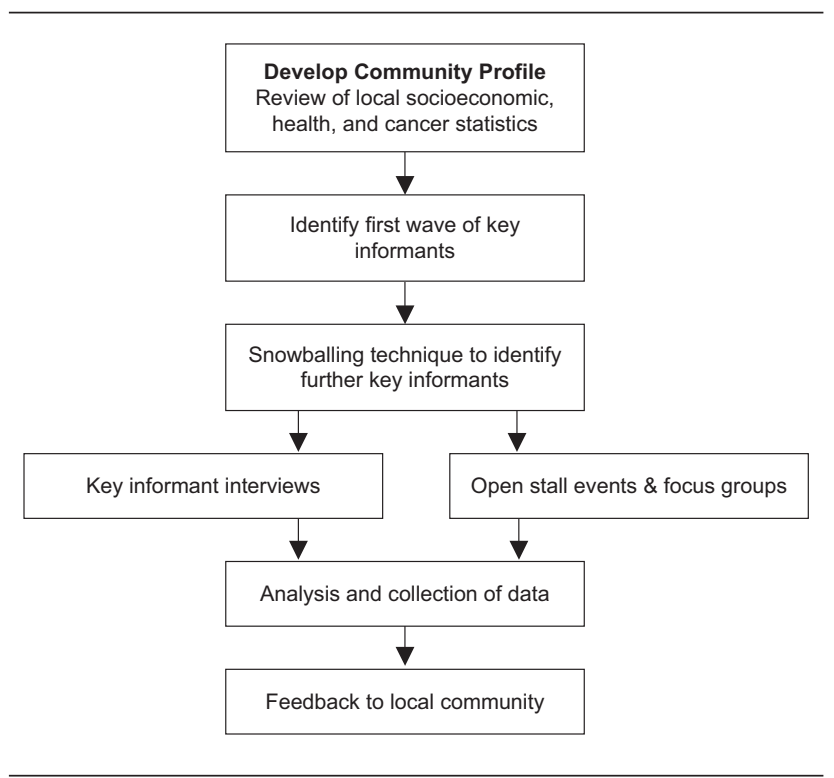

focus groups and key informants, asking the general public questions about their views of cancer and cancer care.

\section{Focus Groups}

Twenty nine focus groups were held, with an average of 4 to 8 participants each. Participants were recruited through community groups and social networks in each area, such as schools, sheltered housing, and groups comprising local businessmen. Questions covered understandings and views of cancer and cancer care, with interpreters used to communicate with participants whose first language was not English, if necessary.

\section{Exit Questionnaires}

A short questionnaire was completed by participants to capture demographic information to ensure a sufficient demographic and socioeconomic spread of participants. They also served to identify whether those who took part had been directly or indirectly affected by cancer.

\section{Collating and Analysis of Data}

Initial data was analyzed thematically in a team. Once general themes had been identified, data was analyzed by one researcher and checked by another. Data were analyzed in stages: (a) each data set was analyzed, (b) data sets were triangulated and analyzed as a whole 
rapid appraisal for each community, and (c) the data was analyzed for Scotland as a whole.

\section{Feedback to Local Community}

Preliminary results were fed back to participants for comments and validation in the form of lay summaries and, in some places - on the request of participantsoral presentations. The lay summary of each area and the final report are also posted on the Cancer Care Research Centre Web site (www.cancercare.stir.ac.uk).

\section{Discussion}

Given the relative novelty of using a rapid appraisal approach in engaging the public we employed the methodological principles identified by Rhodes et al. (1999) to evaluate this study. Evaluation of this study focused on (a) the inductive approach, (b) the focus on triangulation, (c) assessment as an integral part of the response, and (d) priority on participation.

\section{The Inductive Approach}

The first principle, the "inductive approach," "allows assessments to develop in response to practical findings as they emerge" (Rhodes et al., 1999, p. 66) The flexibility inherent in this approach gave us the opportunity to refine the research process across the different locations. Analysis was ongoing throughout the fieldwork to allow emerging themes to be fed back into ongoing data collection. Although the findings of our study will be reported at a later date, two examples will be used to illustrate how developing themes were fed back into data collection. Importantly, they also illustrate that the RA approach can be as good as more traditional qualitative approaches in uncovering lay understandings, in particular in socioeconomically disadvantaged, "hard-to reach" communities from which both examples are drawn. For example, we began to note that although most people recognized that cancer was linked with smoking, this general view was discounted in the face of personal experience in many disadvantaged communities:

\section{A lot of people that never smoke get cancer.}

They say smoking, but I don't know, my father smoked his whole life and lived until he was 85 .

It became apparent that public perceptions of the risk associated with cancer were socially evaluated by these participants (Jamieson, 2003), suggesting that the social components of risk might need to be further explored and understood for public health improvement to be shaped effectively. A follow-up study (as yet unpublished) exploring lay understandings of cancer in socially disadvantaged communities has further explored this aspect of the findings (see www.cancercare.stir.ac.uk for further information).

Another developing line of enquiry, almost exclusively voiced in disadvantaged communities, focused on a growing perception that the government and health professionals were acting conspiratorially to avoid telling the truth about the real causes of cancer:

[I don't trust health professionals] because there's always ... because there's always somebody above them that's tellin' them ... now, you cannae [cannot] ... ye cannae let that be public knowledge.

The evident distrust of politicians in society as a whole and the increasing mistrust of health professionals-particularly doctors-might be an underlying factor to this reported belief and was further explored in subsequent data collection activities.

This kind of inductive process poses specific difficulties for researchers to deal with, such as the sharing of sensitive information within communities. For example, when feeding back to service providers the public's perceptions about the incidence of cancer in a particular community, pressures from service providers to feed back the "true" cancer statistics rather than what the public thought about their communities' rates could potentially have undermined the process. Equally, the sensitivity of talking about current rates of cancer and providing cancer information within particular communities with members of the public was challenging. Providing cancer information was not within the remit of the researchers, yet giving something back is an important part of participatory research processes and a member of staff from a leading cancer information service, Cancerbackup (www.cancerbackup.org.uk), was present at all the open stalls to answer any queries and provide support to participants as necessary.

\section{Triangulation}

The second principle highlighted by Rhodes and colleagues (1999) is 'triangulation between multiple methods and data sources." Using multiple methods and respondents is believed to be a valuable tool to gain a more holistic picture and cross-check findings. This study adopted both multiple methods of research (questionnaires, interviews, focus groups, and market research techniques) and sources of data. The open stalls, a quick market research technique, allowed an 
overview of a large number of local people's views of cancer and cancer care to be obtained. This confirmed that the focus group accounts were indeed more widely held by people who might not have belonged to the community groups normally approached for focus groups. Without the data derived from these focus groups, however, there would have been little depth in the data obtained.

This multisite study was subject to tight deadlines and as such, hit a tension between speed of assessment and depth of community involvement. Although the process of rapid appraisal might be less time-consuming than many other methods, project coordination was logistically difficult and time-consuming. The organization of multisite focus groups, open stalls, and key informant interviews within a tight timeline required good organizational skills and communication within the team, and the ability to develop good relationships with key stakeholders early on. Although we conducted ten rapid appraisals in as many months with a small team, working on different sites at the same time was a challenge to the researchers in remaining focused on the issues arising within one community, as well as maintaining an overview on developing lines of inquiry.

In some locations restricted opportunities to contact groups were offset by opportunities to meet other groups similar in socioeconomic and demographical characteristics in other locations. In this way we achieved a maximum variation sample: a sufficient cross-section of men, women, and children of different ages, ethnicity, socioeconomic circumstances, and experience of cancer to reflect the wide range of views held by the Scottish public.

This large-scale study, which involved over 500 people, generated a wealth of public understandings and perceptions by combining data from across Scotland. This generated a wealth of public health information about cancer and cancer care, but could be as applicable to other health issues where public involvement is desired. Exit questionnaires were used with all participants to ensure we were successful in reaching all the groups identified in our purposeful sampling strategy. In particular we were able to reach those groups traditionally considered hard-to-reach, including children, young people, men, socially-deprived communities, and minority ethnic groups. For example, a third of the focus group participants were from minority ethnic groups. Significantly, the proportion of participants from the general public was seven times higher than the number of professionals, providing a weighting in favor of public perspectives which is not always evident in user involvement studies (Oliver et al., 2004).

\section{Assessment as an Integral Part of the Response}

The third principle identified by Rhodes and colleauges focuses on the outcomes of appraisal and the extent to which the assessment process is "an integral part of the response." Our study was conducted in the first year of a 3-year progam and contributed to the information-gathering phase of the program, alongside a literature review, scoping exercise, and patientinvolvement work. As such, the study provides a baseline of public perspectives which will inform the latter stages of the research program. Further research into the views of members of the public about cancer and health improvement messages has recently been conducted to explore the findings of this study in deprived communities in more detail. A bid to fund an outreach cancer worker, suggested by one of the deprived communities itself, is currently pending. The findings will also be widely disseminated to the public as well as health professionals and academics to maximize the impact on policy, research, and practice.

Against a backdrop of consultation fatigue and cynicism about consultation with no results, it was important that participants did not have unrealistic expectations that involvement in the research would lead directly to changes in services at this stage. However, the public was asked what key messages they had for the Minister of Health and were made aware that this information would be reported to the Scottish Executive Health Department, arguably raising expectations that these messages would be heard and acted upon despite our efforts in explaining our role in informing them, rather than ensuring action.

\section{Priority on Participation}

Finally, Rhodes et al. (1999) identify the "priority given to participatory initiatives" within rapid appraisal. Good community participation takes time, patience, considerable organization, and cooperation with stakeholders. We found much goodwill on the part of the public and professionals in participating in this study. Finding networks was the first step in approaching communities. Fitch et al. (2004) acknowledge the benefits of researchers' prior knowledge and networks, and this was demonstrated in this study by the many and varied connections of the research team across Scotland. It is important not to rely solely on such networks as they could potentially bias outcomes. The Internet provided easy access to community directories, NHS Web sites, and tourist maps of the smaller Scottish towns involved, and all these provided further information about the 
communities, groups, and individuals in the locations identified. The principle of "going where the people were" resulted in a large number of well-attended focus groups; these were mainly held with existing groups in their normal meeting places. They included a men's group in a church where one of the members worked, a parent and toddler group in a community center, and a women's group in one of their homes. Focus groups were based around the discussion of a number of questions about participants understandings, experiences, and perceptions about cancer. No other participatory tools were used and no attempt at consensus or agreeing priorities was made. Findings therefore reflect individual views and were subsequently subjected to analysis by the research team.

In sum, the use of an RA approach was found to provide a sufficiently rigorous framework for meaningful public involvement (for a relevant discussion on rigor in qualitative research, see Davies \& Dodd, 2002). Yet on a continuum of participation, such as that set out by Arnstein (1969), this type of engagement could be characterized as "consultation," rather than full participation or ownership of the process. Members of the communities identified took no part in planning the study or presenting the findings, and had only limited involvement in analyzing the findings during the feedback meetings. There was little information in the final report about the environment people were living in, in terms of their social and cultural contexts. However, this was more a reflection of our approach than the process of rapid appraisal.

\section{Conclusion}

Taking an RA approach provides a useful framework for public involvement in health issues, facilitating consultation which is not exclusively centered on health issues, reflecting the social, political, economic, and physical factors potentially affecting health improvement. It is not, however, an inexpensive or particularly easy option. It demands time and resources to work effectively and is dependent on real engagement with communities. Conducted in this way, it is not a "quick and dirty," but rapid and robust way of conducting research or engaging the public.

Future studies aiming to contribute to policy and planning for cancer care and health improvement by involving the public would further our RA approach from robust consultation to meaningful participation by involving members of the public throughout the research process, and by creating immediate policy and/or practical change. Through this article we hope to have demonstrated that members of the general public are very willing to discuss health and health care, and that no group is "hard to reach" if engagement is on their terms.

\section{References}

Arnstein, S. (1969). A ladder of participation. Journal of the American Institute of Planners, 35(4), 216-224.

Burrows, D., Trautmann, F., Bijl, M., \& Sarankov, Y. (1999). Training in the Russian Federation on rapid assessment and response to HIV/AIDS among injecting drug users. Journal of Drug Issues, 29(4), 811-842.

Campbell, J. (2002). A critical appraisal of participatory methods in development research International Journal of Social Research Methodology, 5(1), 19-29.

Cornwall, A., \& Guijt, I. (2004). Shifting perceptions, changing practices in PRA: From infinite innovation to the quest for quality. Participatory Learning and Action, 50, 160-167.

Davies, D., \& Dodd, J. (2002). Qualitative research and the question of rigor. Qualitative Health Research, 12, 279-289.

Dorabjee, J., \& Samson, L. (2000). A multi-centre rapid assessment of injecting drug use in India. International Journal of Drug Policy, 11, 99-112.

Fitch, C., Stimson, G. V., Rhodes, T., \& Poznyak, V. (2004). Rapid assessment: An international review of diffusion, practice and outcomes in the substance use field. Social Science and Medicine, 59, 1819-1830.

Heaver, R. (1992). Participatory rural appraisal: Potential application in family planning, health and nutrition programmes. RRA Notes Number 16: Special Issue on Application for Health, 13-21.

Jamieson, W. V. (2003). Risk as a social phenomenon. Forum of Nutrition, 56, 327-330.

Koelen, M., Vaandrager, L., \& Colomer, C. (2001). Health promotion research: Dilemmas and challenges. Journal of Epidemiology and Community Health, 55, 257-262.

MacDougall, C., \& Fudge, E. (2001). Planning and recruiting the sample for focus groups and in-depth interviews. Qualitative Health Research, 11, 117-126.

Manderson, L., \& Aaby, P. (1992). An epidemic in the field? Rapid assessment procedures and health research. Social Science and Medicine, 35, 839-850.

Market Research Society/Social Research Association (2005, October). Data Protection Act 1998: Guidelines for social research. Retrieved March 31, 2008 from www.mrs.org.uk/ standards/downloads/revised/legal/data_protection_social.pdf

Materia, E., Imoko, J., Berhe, G., Dawuda, C., Omar, M. A., Pinto, A., et al. (1995). Rapid surveys in support of district health information systems: An experience from Uganda. East African Medical Journal, 72(1), 15-18.

McKeganey, N. (2000). Rapid assessment and response: Really useful knowledge or an argument for bad science? International Journal of Drug Policy, 11, 13-18.

Murray, S. (1999). Experiences with "rapid appraisal" in primary care: Involving the public in assessing health needs, orienting staff, and educating medical students. British Medical Journal, 318, 440-444. 
National Health Services Scotland, Information Services Division (2007). Cancer in Scotland 2007. Retrieved March 31, 2008, from www.isdscotland.org/isd.scot.nhs.uk/.../FileBuffer?namedFile= Cancer_in_Scotland_summary_m.pdf\&pContentDispositionType= inline

Needle, R. H., Trotter, R. T., Singer, M., Bates, C., Page, J. B., Metzger, D., \& Marcelin, L. H. (2003). Rapid assessment of the HIV/AIDS crisis in racial and ethnic minority communities: An approach for timely community interventions. American Journal of Public Health, 93, 970-979.

Oliver, S., Clarke-Jones, L., Rees, R., Milne, R., Buchanan, P., Gabbay, J., et al. (2004). Involving consumers in research and development agenda setting for the NHS: Developing an evidencebased approach. Health Technology Assessment, 8(15), 1-148.

Ong, B. N., \& Humphries, G. (1994). Prioritizing needs with communities: Rapid appraisal methodologies in health. In J. Popay \& J. Williams (Eds.), Researching the People's Health. London: Routledge.

Paredes, S., Estada, R., Alarcon, H., Chavez, G., Romero, M., \& Hay, R. (1997). Can school teachers improve the management and prevention of skin disease? A pilot study based on head louse infestations in Guerrero, Mexico. International Journal of Dermatology, 36(11), 826-830.

Rhodes, T., Stimson, G., Fitch, C., Ball, A., \& Renton, A. (1999). Rapid assessment, injecting drug use and public health. Lancet, 354, 65-68.
Scottish Executive Health Department (2000). Improving health in Scotland-The challenge. Retrieved August 15, 2006, from http://www.scotland.gov.uk/Publications/2003/03/16747/19929

Scottish Executive Health Department (2005). Delivering for health. Edinburgh: Author.

Tones, K., \& Green, J. (2004). Health promotion: Planning and strategies. London: Sage.

Neneh Rowa-Dewar, MSc, is a research fellow at the Cancer Care Research Centre, University of Stirling in Stirling, Scotland.

Wendy Ager, MSc, is a research assistant at the Cancer Care Research Centre, University of Stirling in Stirling, Scotland.

Katherine Ryan, $\mathrm{PhD}$, is a research fellow at the Cancer Care Research Centre, University of Stirling in Stirling, Scotland.

Irene Hargan, MSc, is a research assistant at the Cancer Care Research Centre, University of Stirling in Stirling, Scotland.

Gill Hubbard, $\mathrm{PhD}$, is a senior research fellow at the Cancer Care Research Centre, University of Stirling in Stirling, Scotland.

Nora Kearney, RGN MSc, is a professor at the Cancer Care Research Centre, University of Stirling in Stirling, Scotland. 